\title{
Elliptic st. jude bileaflet mechanical heart valves
}

\author{
Hadi Mohammadi ${ }^{1,2^{*}}$, Mehdi Jahandardoost ${ }^{1}$ and Guy Fradet ${ }^{3}$ \\ *Correspondence: hadi.mohammadi@ubc.ca

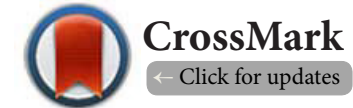

'School of Engineering, Faculty of Applied Science, University of British Columbia, Kelowna, BC, Canada.

${ }^{2}$ Faculty of Applied Science, University of British Columbia, Vancouver, BC, Canada.

${ }^{3}$ Department of Surgery, Faculty of Medicine, University of British Columbia, Vancouver, BC, Canada.

\begin{abstract}
St. Jude Medical (SJM) bileaflet mechanical valves were approved by the Food and Drug Administration in 1977. The SJM valve design consists of two semicircular leaflets which pivot on hinges. Compared to other mechanical heart valve prostheses such as ball and cage and tilting disk prosthetic valves, it provides good central flow, the leaflets open completely, and the pressure drop across the valve is trivial. However, non-physiological hemodynamics around these valves may lead to red blood cells lysis and therombigenic complications. Also, the regurgitation-flow inSJM valves is almost twice that of the native valves in the aortic position. In this study, we suggest a new design for the stent (housing) of SJM valves in which $15 \%$ ovality is applied to the stent whereas its perimeter remains constant. In a pilot study, the hemodynamic performance of the proposed design is analyzed in the closing phaseand compared to that of conventional SJM models. Results show that while the elliptic SJM model offers a shorter closing phase (9.7\% shorter), the regurgitation flow remains almost unchanged. In other words, even though the dynamic response of the valve is improved, the regurgitation flow is not decreased. Thus, a more efficient effective orifice area (EOA) is shown to be provided by the proposed model. The preliminary calculations presented in this study justify an improved hemodynamics of elliptic SJM valves compared to conventional models; the proposed design shows promise and merits further development.

Keywords: Finite strip method, vascular hemodynamics, bileaflet mechanical heart valves, st. jude medical valve, heart valve prostheses, numerical modeling
\end{abstract}

\section{Introduction}

Bileaflet mechanical heart valves (MHVs) are used extensively due to their great hemodynamic performance, as indicated by a uniform flow profile, free central flow and considerably higher level of durability. However, thrombogenicity is an issue with the bileaflet MHVs due to a non-physiological hemodynamics around the valve [1-3]. More than 300,000 replacement heart valves are implanted annually worldwide and mechanical heart valves are used to replace diseased human heart valves in approximately $50 \%$ of these interventions. In addition, two million patients receive St. Jude Medical (SJM) valves worldwide each year $[4,5]$. In our previous studies, we extensively assessed the hemodynamic performance of the SJM valve in the opening phase using computational fluid dynamics (CFD). Results suggested that SJM valves may be associated with thrombogenic complications around the hinges, on the leading edge of the leaflets and at the sinuses, possibly because of high blood shear stresses, turbulence, and the overall complexity of the hemodynamics in MHVs [6,7].

In this study, we propose a design modification on SJM valves. We hypothesize that $15 \%$ ovality on the housing while its perimeter remains constant may result in an improved hemodynamics around the SJM valves. In a pilot study, we study the hemodynamic performance of the proposed design to evaluate its regurgitation flow and its velocity and the leaflet tip velocity in the closing phase. We apply a quick but novel numerical model which is sufficiently accurate to estimate the overall performance of the new design. The numerical model uses the finite strips method to solve the equations of motion. The computations run on an Intel (R) Core (TM) i7-4500u CPU @1.80 GHz \& $1.80 \mathrm{GHz}$ processors with $16.0 \mathrm{~Gb}$ of RAM.

\section{Methods}

The SJM model considered in this study has an inner diameter 
Mohammadi et al. Cardiovascular System 2015,

of $25 \mathrm{~mm}$ as shown in Figures $\mathbf{1} \mathbf{a}$ and $\mathbf{1} \mathbf{b}$. In the proposed design, the perimeter of the housing remains constant. The housing is elliptic with a major diameter of $27 \mathrm{~mm}$ and a minor diameter of $23 \mathrm{~mm}$ as shown in Figures $1 \mathrm{c}$ and $1 \mathrm{~d}$. Due to the leaflet's rotation, the computational domain is defined as a control volume (CV) with moving boundaries as shown in Figures $\mathbf{2 a}$ and $\mathbf{2} \mathbf{b}$. In order to simplify the computational process, flow is assumed to be unidirectional in the direction of the major axis and the projected area between the leaflets and the aortic wall is assumed to be rectangular at various positions during the closing phase [5]. The flow is considered inviscidsuch that velocity is uniform at every cross section in the CVand the inlet $[\mathbf{8 , 9}$. The regurgitation flow is divided into two regimes through the minor and major orifices at the entrance of each section. When the valve is fully open, it is assumed that the aortic pressure $\left(P_{\mathrm{ao}}\right)$ and the ventricular pressure $\left(P_{v}\right)$ are both uniform and equal. This is because the valve remains fully open during the systole with almost no forward flow. The control volume is $A B C D$, where $A B$ is the leaflet, $\mathrm{o}$ is the pivot, and $\mathrm{EF}$ is an arbitrary section. $\mathrm{U}_{A D^{\prime}} \mathrm{U}_{B C^{\prime}}$ and $U_{E F}$ are the velocities of blood at the entrance (AD), at the outlet (BC) and at the arbitrary section (EF), respectively. The distance from the EF section with respect to the inlet section is denoted as $Y . I_{A D^{\prime}} I_{b c}$ and $I_{E F}$ are the length of the inlet and the outlet and the arbitrary sections which are time dependent but their width (w) remains constant (Figure 2). Velocities and pressures vary constantly through the sections $A D$ to $B C$. Mass is conserved within the CV such that the velocity at the $\mathrm{EF}$ section $\left(\mathrm{V}_{\mathrm{EF}}\right)$ is calculated with respect to the inlet velocity $\left(\mathrm{V}_{\mathrm{AD}}\right)$ or the outlet velocity $\left(\mathrm{V}_{\mathrm{BC}}\right)$ in the $\mathrm{CV}$. It should be noted that the velocity of blood in the vicinity of the leaflet tips and at the EF section is higher than the axial velocity of the leaflet tip $\left(\mathrm{V}_{\mathrm{tAD}} \mathrm{V}_{\mathrm{tBC}}\right)$ and the axial velocity of the leaflet at the EF section $\left(\mathrm{V}_{\mathrm{tEF}}\right)$, respectively. The unsteady continuity equation on the $\mathrm{CV}$ takes the form:

$$
A_{A D}\left(V_{A D}-V_{t A D}\right)=A_{E F}\left(V_{E F}-V_{t E F}\right)+\frac{d V i}{d t}
$$

where $A_{A D}\left(w I_{A D}\right)$ and $A_{E F}\left(W I_{E F}\right)$ are cross sectional areas at the sections $A D$ and $E F$, respectively. The axial velocities of

the leaflet at $\mathrm{A}$ and $\mathrm{E}$ are $V_{t A D}=(r-l) \omega \cos \theta$ and

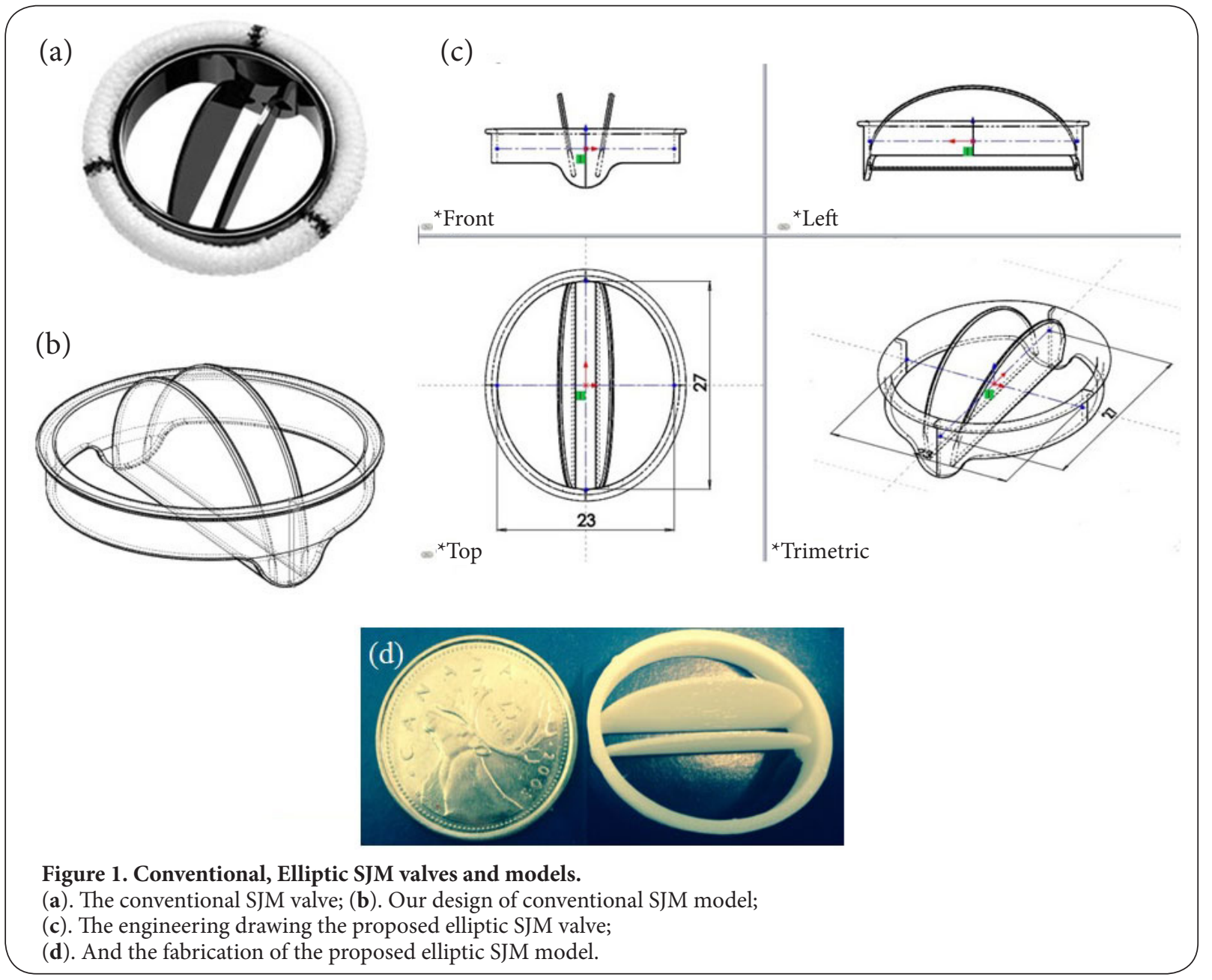




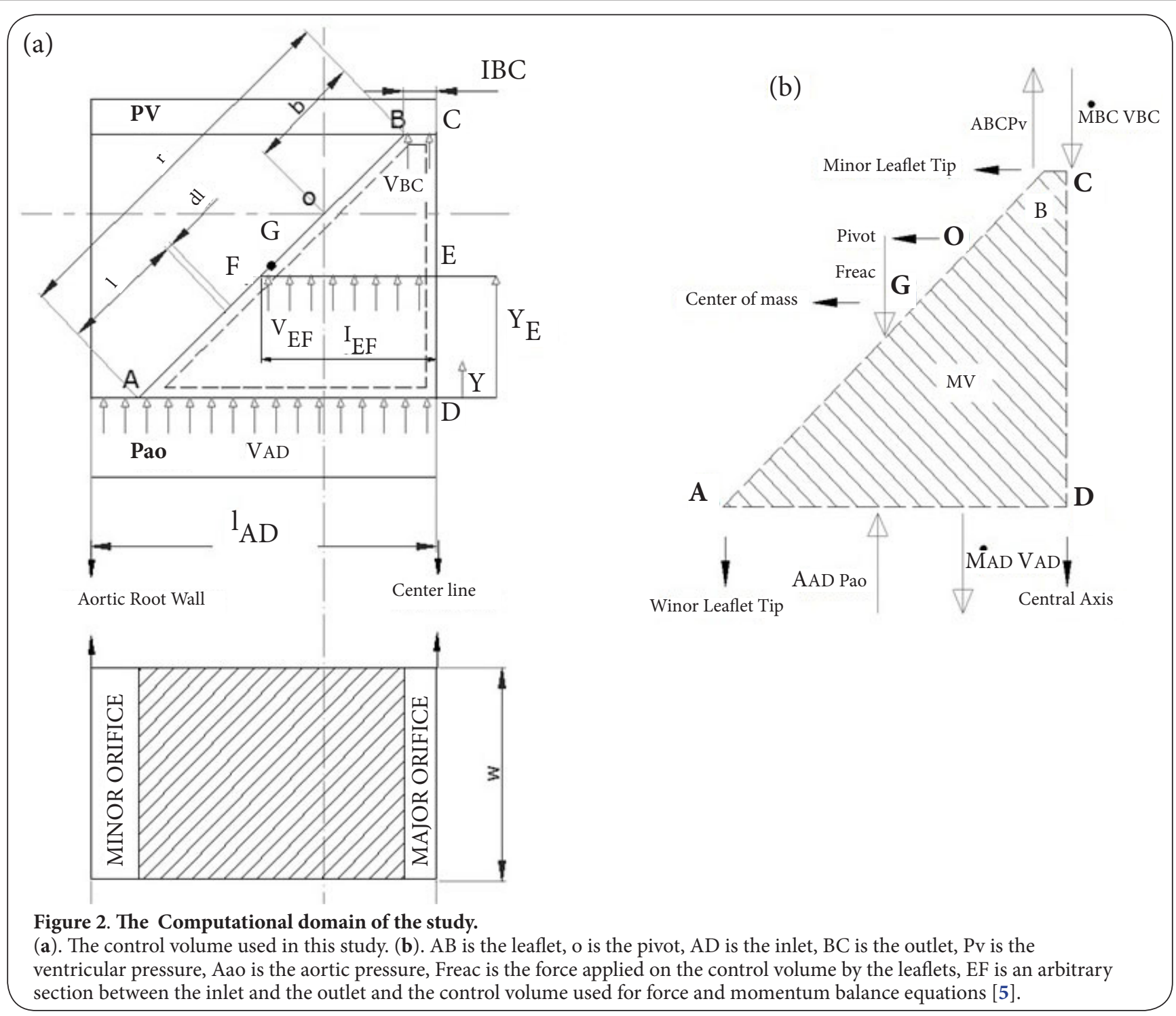

$V_{t E F}=(r-b-l) \omega \cos \theta$, where $r$ is the radius of

the leaflet in the circular SJM valve and the major radius of the leaflet in the elliptic SJM valve. I is the axial distance from the pivot to the major leaflet tip, $b$ is the axial distance of the pivot to the minor leaflet tip, $\omega$ is the angular velocity of the leaflet, and $\theta$ is the angle between the $\mathrm{AD}$ and the leaflet. $\mathrm{V}_{\mathrm{i}}$ is the volume of the $\mathrm{CV}$ which is $V_{i}=\left(l_{A D}+l_{E F}\right) w s \sin \theta / 2$. Applying the principle of the conservation of momentum on the CV gives:

$$
\begin{aligned}
& \left(A_{A D} P_{a o}-A_{B C} P_{v}-F_{\text {reac }}\right)-\left(\dot{m}_{B C} V_{B C}-\dot{m}_{A D} V_{A D}\right) \\
& =d(M V) / d t
\end{aligned}
$$

where $A_{B C}\left(\mathrm{wl}_{B C}\right)$ is the cross sectional area at the outlet, $\bar{m}_{B C}$ is the mass flow at the outlet which is

$$
d m_{B C}=\rho A_{B C}\left(V_{V C}-V_{t B C}\right) d t
$$

where $\rho$ is the density of blood and $U_{t B C}=-b \omega \cos \theta$,

$\dot{m}_{A D}$ is the mass flow in the inlet which is

$$
d m_{A D}=\rho A_{A D}\left(V_{A D}-V_{t A D}\right) d t
$$

where $\mathrm{dt}$ is the time increment, $F_{\text {reac }}$ is the axial force applied on the $C V$ by the leaflet, $M$ is the mass of the $C V$ which is

$M=\rho w d\left(l_{A D}+l_{B C}\right) \sin \theta / 2$ where $\mathrm{d}$ is the axial distance between the inlet and the outlet, and $\mathrm{V}$ is the velocity of the $\mathrm{CV}$ which is $V=\left(V_{A D}+V_{B C}\right) / 2$. Using the Eq. 2, $F_{\text {reac }}$ is calculated.

Also, the unsteady energy equation between the inlet and the outlet is applied to calculate $F_{\text {reac }}$ such that: 
Mohammadi et al. Cardiovascular System 2015,

$\left(\frac{V_{A D}^{2}-V_{E F}^{2}}{2}\right)+\left(\frac{P_{A D}-P_{E F}}{\rho}\right)=\int_{0}^{y_{E F}}(d V / d t) d y$

where $P_{A D}$ is the aortic pressure and $P_{E F}$ is the pressure at the section EF. $Y_{E F}$ is the axial distance between $A D$ and FE.

Assuming $(d V / d t)$ as:

$$
\frac{\partial V}{\partial t}=\left[\left(V_{j}\right)_{t}-\left(V_{j}\right)_{t-\delta t}\right] / \delta t
$$

Then the term of the right part of equation is expressed:

$$
\int_{o}^{y_{m}}(d V / d t) d y=\sum_{j=1}^{N}\left[\left(V_{j}\right)_{t}-\left(V_{j}\right)_{t-\delta t}\right] \delta l \sin \theta / \delta t
$$

where the subscript " $t$ ", "t$t-\delta t$ " represents the value at time $t$, and $\mathrm{t}-\delta \mathrm{t}$, respectively, $\delta \mathrm{t}$ is the time increment and $\delta l=\frac{r}{N}$

where $\mathrm{N}$ is the number of strips. The set of equations of 3 to 5 gives the pressure at an arbitrary section $\left(\mathrm{P}_{\mathrm{EF}}\right)$ which is used to calculate $F_{\text {reac }}$ :

$$
F_{\text {reac }}=\int^{r} w P_{E F} \cos \theta d l=\sum_{m=1}^{N} w \delta l \cos \theta P_{m} .
$$

The two approaches used to calculate $F_{\text {reac }}$ are solved together simultaneously in order to calculate the inlet velocity $\left(\mathrm{V}_{\mathrm{AD}}\right)$. Using $\mathrm{V}_{\mathrm{AD}}$ the angular velocity of the leaflets is calculated. The governing equation of motion for the leaflets takes the form:

$$
T_{p}+T_{g}=I_{o}\left(d^{2} \theta / d t^{2}\right) \text { where Tp is the torque }
$$
due to pressure, $\mathrm{Tg}$ is the torque due to gravity, and $\mathrm{I}_{\mathrm{o}}$ is the mass momentum of inertia of the leaflets about the pivot. Tp and $\mathrm{Tg}$ are calculated as such:

$$
\begin{aligned}
& T_{p}=-\int_{N}^{r}\left(P_{E F}-P_{v}\right) w(r-b-l) d s \\
& =\sum_{m=1}^{N}\left(P_{E F}-P_{v}\right) w(r-b-l) \delta s \\
& T_{g}=m_{e} g(r / 2-b) \cos \theta
\end{aligned}
$$

where $m_{e}=A_{o}^{\prime} \Delta\left(\rho_{o}-\rho\right)$ is the equivalent mass of the leaflets by taking the buoyant force into account. Á and $\Delta$ are the area and the thickness of each leaflet and $\rho_{0}$ is the density of the leaflets. The two necessary initial conditions are based on the maximum opening angle of the SJM valves which is $\theta(0)=85^{\circ}$ and the velocity at the beginning of the closing phase is considered $0 \mathrm{~m} / \mathrm{s}$. The regurgitation flow is calculated by $Q_{f f}=A_{o} V_{i} \delta t$ where $Q_{r f}$ is the regurgitation flow and $\mathrm{A}_{\text {or }}$ is the total orifice area.

\section{Results}

The hemodynamic performance of proposed design including the velocity of the blood and the velocity of the leaflets and the regurgitation flow volume are calculated with respect to time in the beginning of the diastolic phase, i.e., the closing phase. The time increment $\delta \mathrm{t}$ is chosen to be $0.05 \mathrm{~ms}$. Also, mentioned above, $F_{\text {reac }}$ is calculated using the two approaches in order to calculate the inlet velocity. The converging criterion specified is that the calculated $F_{\text {reac }}$ from the two approaches should be less than or equal to $0.001(\mathrm{~N})$. When the leaflet angle is less than 1 degree, it is assumed that the leaflet is very close to the final closed position. Time, $t$, is selected to be zero at the moment where backflow is initiated and it is assumed that the initial velocity of the fluid is zero. The leaflet is divided into 30 strips of equal width. The governing equations of motion for the leafletare solved by fourth order Runge-Kutta method. The heart rate and cardiac output were selected to be 70 beats per minute $(\mathrm{bmp})$ and 6 lit/min, respectively [10]. The ventricular pressure is assumed to decrease from a value equal to the aortic pressure (when the valve is fully opened) and the average aortic pressure is considered to be $16.0 \mathrm{kPa}$, i.e., $120 \mathrm{mmHg}$ in the closing phase and is assumed to be constant all along.

The velocity of the leaflet tip, the velocity of the blood flow in the vicinity of the leaflet tip and the regurgitation flow volume of the conventional SJM valves obtained in this study and those reported before $[5,11]$ are consistent. Figure 3 shows the velocity of the leaflet tip in the closing phase for both designs. The velocity of the leaflet tip in the elliptic design is higher than that of the conventional design. It also shows that the closing phase in the elliptic design is $9.7 \%$ lower. Figure 4 shows the velocity of the regurgitation flow in the vicinity of the leaflet tip for the two elliptic and conventional SJM valves. The velocity of the regurgitation flow in the elliptic model shows an average increase of $11 \%$ compared to the conventional SJM valve. The higher velocity of the regurgitation flow in the elliptic SJM model leads to a shorter closing phase. The two effective parameters to

\section{Axial velocity of the leaflet tip $[\mathrm{m} / \mathrm{s}]$}

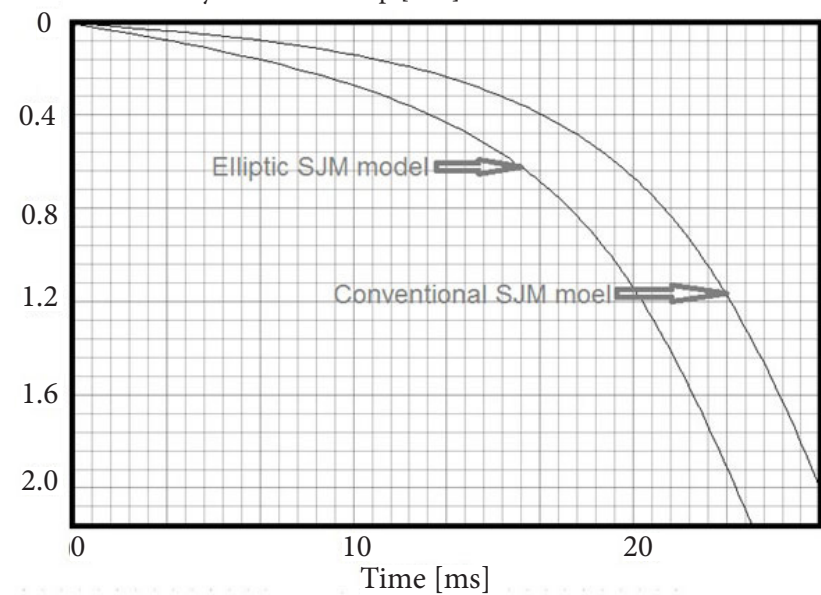

Figure 3. The velocity of the leaflet tip in the closing phase for both elliptic and conventional SJM valves. 
Mohammadi et al. Cardiovascular System 2015,

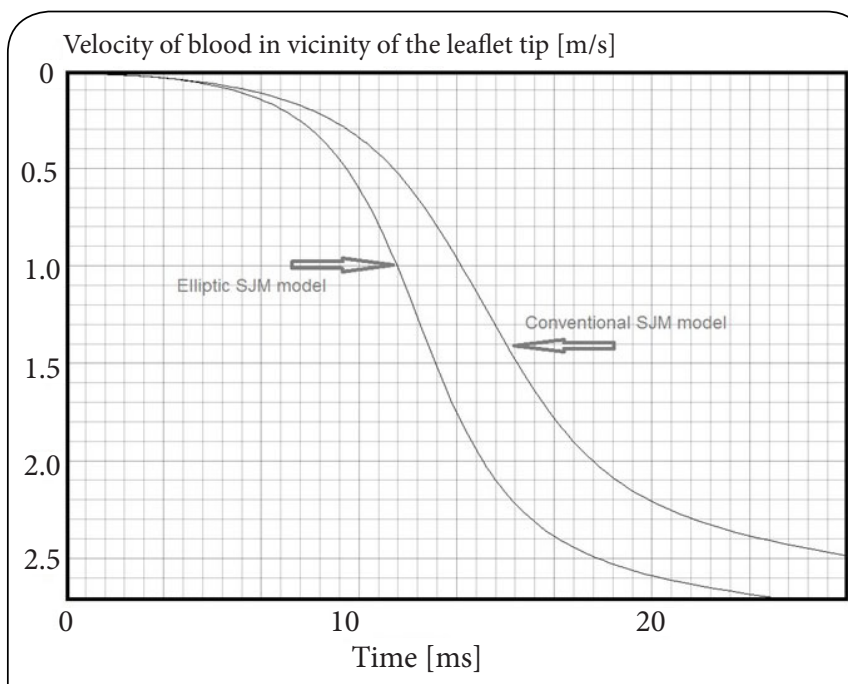

Figure 4. The velocity of the regurgitation flow in the vicinity of the leaflet tip in the closing phase for both elliptic and conventional SJM valves.

calculate the regurgitation flow volume is (1) the closing phase time and (2) the velocity of the blood which is shown in Figure 5. Results show that even though the velocity of the regurgitation flow in the elliptic SJM model is higher than that of the conventional SJM model, the backflow volume in the two models is comparable and equal $(<0.05 \%$ error).

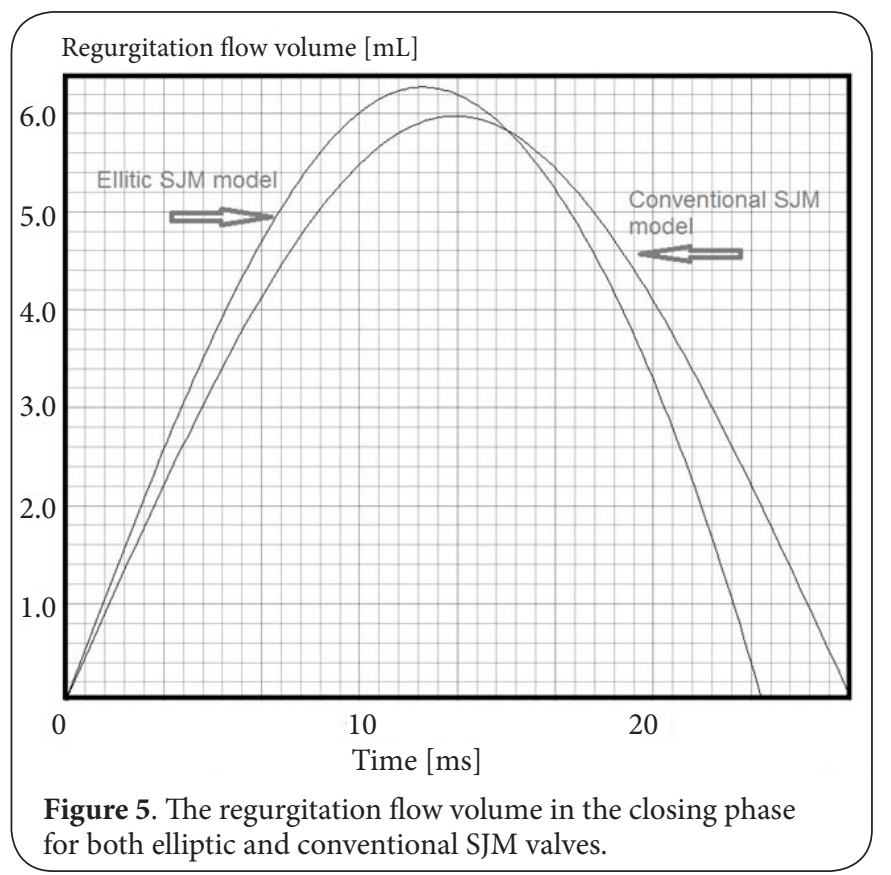

\section{Conclusion}

In this study, we proposed a design modification to the SJM conventional valves and developed a numerical tool that can quickly assess the hemodynamic performance of bileaflet mechanical heart valves in general and the elliptic SJM valve proposed in this study in particular. Results of the current study suggest a clear improvement in the hemodynamic performance of elliptic SJM valves over the conventional models. A comprehensive set of experimental and computational studies in the opening and closing phases will further address the hemodynamic performance of the proposed elliptic SJM valve.

\section{Competing interests}

The authors declare that they have no competing interests.

\section{Authors' contributions}

\begin{tabular}{|l|c|c|c|}
\hline Authors' contributions & HM & MJ & GF \\
\hline Research concept and design & $\checkmark$ & -- & -- \\
\hline Collection and/or assembly of data & $\checkmark$ & $\checkmark$ & $\checkmark$ \\
\hline Data analysis and interpretation & $\checkmark$ & $\checkmark$ & -- \\
\hline Writing the article & $\checkmark$ & $\checkmark$ & -- \\
\hline Critical revision of the article & $\checkmark$ & -- & $\checkmark$ \\
\hline Final approval of article & $\checkmark$ & -- & $\checkmark$ \\
\hline Statistical analysis & $\checkmark$ & $\checkmark$ & -- \\
\hline
\end{tabular}

\section{Acknowledgement and funding}

The authors would like to thank the University of British Columbia and the NSERC/DG for financially supporting this study. Start-up Grant, University of British Columbia.

\section{Publication history}

Senior Editor: Shiwei Duan, Ningbo University, China.

Received: 12-Jan-2015 Final Revised: 13-Feb-2015

Accepted: 02-Mar-2015 Published: 09-Mar-2015

\section{References}

1. Mohammadi $\mathrm{H}$ and Mequanint $\mathrm{K}$. Prosthetic aortic heart valves: modeling and design. Med Eng Phys. 2011; 33:131-47. | Article | PubMed

2. Mohammadi $\mathrm{H}$, Bahramian $\mathrm{F}$ and Wan W. Advanced modeling strategy for the analysis of heart valve leaflet tissue mechanics using high-order finite element method. Med Eng Phys. 2009; 31:1110-7. | Article | PubMed

3. Mohammadi H, Klassen RJ and Wan WK. A finite element model on effects of impact load and cavitation on fatigue crack propagation in mechanical bileaflet aortic heart valve. Proc Inst Mech Eng H. 2008; 222:1115-25. | Article | PubMed

4. Mohammadi H, Boughner D, Millon LE and Wan WK. Design and simulation of a poly(vinyl alcohol)-bacterial cellulose nanocomposite mechanical aortic heart valve prosthesis. Proc Inst Mech Eng H. 2009; 223:697-711. | Article | PubMed

5. Mohammadi $\mathrm{H}$, Ahmadian MT and Wan WK. Time-dependent analysis of leaflets in mechanical aortic bileaflet heart valves in closing phase using the finite strip method. Med Eng Phys. 2006; 28:122-33. | Article I PubMed

6. Jahandardoost M, Fradet G and Mohammadi H. A New Computational Model for the Hemodynamics of Bileaflet Mechanical Valves in the Opening Phase. Proceeding of the institution in mechanical engineering Part H. J of Eng. in Medicine. 2014.

7. Jahandardoost M, Fradet $\mathrm{G}$ and Mohammadi H. Effect of Pulsatility Rate 
Mohammadi et al. Cardiovascular System 2015,

http://www.hoajonline.com/journals/pdf/2052-4358-3-1.pdf

on the Hemodynamics of Bileaflet Mechanical Prosthetic Heart Valves (St. Jude Medical Model) for the Aortic Position in the Opening Phase; A Computational Study. J of Cardiovascular Engineering and Technology. 2014.

8. Reif TH. A numerical analysis of the backflow between the leaflets of a St Jude Medical cardiac valve prosthesis. J Biomech. 1991; 24:733-41. | Article | PubMed

9. van Steenhoven AA, van Duppen TJ, Cauwenberg JW and van Renterghem RJ. In vitro closing behaviour of Bjork-Shiley, St Jude and Hancock heart valve prostheses in relation to the in vivo recorded aortic valve closure. J Biomech. 1982; 15:841-8. I Article I PubMed

10. Mohammadi H and Mequanint K. An Inverse Numerical Approach for Modeling Aortic Heart Valve Leaflet Tissue Oxygenation. Journal of Cardiovascular Engineering and Technology. 2011; 3:73-79. | Article

11. Subramanian A, Mu H, Kadambi JR, Wernet MP, Brendzel AM and Harasaki $H$. Particle image velocimetry investigation of intravalvular flow fields of a bileaflet mechanical heart valve in a pulsatile flow. $J$ Heart Valve Dis. 2000; 9:721-31. I Article I PubMed

\section{Citation:}

Mohammadi H, Jahandardoost M and Fradet G.

Elliptic st. jude bileaflet mechanical heart valves.

Cardio Vasc Syst. 2015; 3:1.

http://dx.doi.org/10.7243/2052-4358-3-1 